La revue La revue pour l'histoire du CNRS

POUR LHISTORE DU CNRS $\quad 20 \mid 2008$

Aventures et recherches aux pôles

IPN : un cyclotron pour les ions lourds

De Gatlinburg à la Plagne (1958-1969)

René Bimbot

CpenEdition

Journals

Édition électronique

URL : https://journals.openedition.org/histoire-cnrs/6492

DOI : $10.4000 /$ histoire-cnrs. 6492

ISSN : 1955-2408

Éditeur

CNRS Éditions

Édition imprimée

Date de publication : 3 avril 2008

ISBN : 978-2-271-06562-9

ISSN : 1298-9800

Référence électronique

René Bimbot, "IPN : un cyclotron pour les ions lourds », La revue pour l'histoire du CNRS [En ligne], 20 | 2008, mis en ligne le 03 avril 2010, consulté le 20 mai 2021. URL : http://journals.openedition.org/ histoire-cnrs/6492 ; DOI : https://doi.org/10.4000/histoire-cnrs.6492

Ce document a été généré automatiquement le 20 mai 2021

Comité pour l'histoire du CNRS 


\section{IPN : un cyclotron pour les ions lourds}

De Gatlinburg à la Plagne (1958-1969)

\section{René Bimbot}

1 En mai 1958, avant même que le Synchrocyclotron à protons, premier grand accélérateur du Laboratoire de physique nucléaire d'Orsay ait délivré le moindre faisceau, se tient à Gatlinburg, station de montagne du Tennessee, non loin du grand centre nucléaire d'Oak- Ridge, la première conférence de l'histoire concernant les réactions induites par ions lourds [1]. Ce terme désigne les projectiles de masse supérieure à celle des isotopes de l'hélium, qui avec le proton et le deuton, constituent les projectiles dits "légers ». Les ions lourds les plus courants à cette époque sont le carbone $12\left({ }^{12} \mathrm{C}\right)$ et l'azote $14\left({ }^{14} \mathrm{~N}\right)$. Bien qu'un faisceau de carbone ait été obtenu dans un cyclotron de Berkeley dès 1940 1, les machines capables d'accélérer de tels projectiles dans de bonnes conditions se comptent encore sur les doigts d'une main : deux cyclotrons d'environ 1,50 $\mathrm{m}$ de diamètre ${ }^{2}$, l'un américain (Oak-Ridge), l'autre soviétique (Moscou), et un accélérateur linéaire à Berkeley ${ }^{3}$. Il existe également quelques machines circulaires construites pour accélérer des particules légères et aménagées pour les ions lourds, mais qui fournissent des faisceaux de faible intensité et dont l'énergie est très mal définie.

2 Ainsi, à Saclay, un cyclotron de $160 \mathrm{~cm}$ délivre des faisceaux d'azote et d'oxygène ${ }^{4}$ depuis 1956. À l'initiative d'Irène Joliot-Curie, un cyclotron spécialement dédié aux ions lourds a été prévu dès 1955 parmi les futurs accélérateurs du Laboratoire de physique nucléaire d'Orsay. Ses caractéristiques ont été définies en 1957 et, la même année, l'électro-aimant qui en constitue la pièce maîtresse a été commandé par Frédéric Joliot. Cette machine, qui se distingue des accélérateurs à protons ou à deutons par un champ magnétique et une haute fréquence variables, permettant d'adapter les conditions d'accélération à une large gamme d'ions, sera le «Cyclotron à énergie variable à ions lourds ", d'abord abrégé en "CEVIL », puis, plus tard en "CEV»[2]. Mais quelles raisons poussent physiciens et chimistes à s'intéresser à ces nouveaux projectiles?

L'intérêt des ions lourds 
3 Tous les physiciens nucléaires ne sont pas favorables à l'utilisation de faisceaux d'ions lourds. Certains préfèrent s'en tenir à des projectiles simples, tel le proton, dont la collision avec un noyau conduit, en principe à des phénomènes plus faciles à interpréter. Cependant, les partisans de ces nouveaux projectiles mettent en avant le fait qu'ils sont les seuls à permettre l'accès à toute une gamme de processus inédits. Le plus prévisible d'entre eux, dont l'existence a rapidement été démontrée par les premières expériences, est la possibilité de fusion de ces projectiles avec les noyaux/ cibles, qui conduit à des noyaux - souvent inconnus -, de masses beaucoup plus élevées. Les ions lourds constituent donc un remarquable outil pour la synthèse de ces nouvelles espèces, en particulier dans la région des éléments transuraniens ${ }^{5}$. L'étude du processus de fusion lui-même, et celle de la désexcitation des noyaux qui en résultent, dont la caractéristique essentielle est de posséder une grande énergie de rotation, constituent également des thèmes de recherche très attrayants.

Enfin, les ions lourds induisent d'autres types de réactions (transferts de nucléons, diffusion inélastique) qui présentent un grand intérêt, du point de vue expérimental et théorique ${ }^{6}$. En cette année 1958, les ions lourds ouvrent donc un immense champ de recherche encore presque inexploré.

La construction du CEV

5 Le groupe d'étude initial, composé de chercheurs et d'ingénieurs, comprenait une dizaine de personnes. La direction scientifique de la construction de la machine fut confiée à Marc Lefort et sa réalisation à un groupe d'ingénieurs et de techniciens dont le nombre augmenta régulièrement avec les années, et qui prit le nom de "Service d'électronique pour accélérateurs", sous la responsabilité d'André Cabrespine. Il fut largement fait appel aux autres services techniques du laboratoire et notamment au Bureau d'études et au service de mécanique.

6 L'aimant principal fut livré dès 1959. Des pièces métalliques furent ajoutées de façon à moduler le champ magnétique entre ses pôles ${ }^{7}$ et les mesures débutèrent immédiatement pour établir une cartographie de ce champ dans tout son entrefer. Cependant, pour atteindre la précision souhaitée, il fallut développer des sondes adaptées. De plus, les mesures durent être interrompues pendant près d'un an en raison d'un court-circuit survenu dans l'une des bobines.

7 Six années furent nécessaires pour mener à bien la construction du cyclotron à énergie variable. Il faut dire qu'il ne s'agissait pas moins que de concevoir et de réaliser un accélérateur entièrement original dans un laboratoire lui-même en voie de constitution!

On prend conscience de l'ampleur de cette tâche lorsque l'on sait qu'au-delà de l'aimant principal, chacun de ses éléments - source d'ions plusieurs fois chargés, tels $\mathrm{C}^{4+}$ ou $\mathrm{N}^{4+}$, chambre d'accélération sous vide, système de génération de la tension de haute fréquence accélératrice, déflecteur électrostatique servant à extraire le faisceau de la machine - fit l'objet d'une étude spécifique, comportant la plupart du temps la réalisation d'une maquette ou d'un prototype. Il fallut aussi leur ajouter divers accessoires majeurs tels que l'ensemble de pompage, capable de réaliser un vide poussé dans tout le volume de la chambre d'accélération et des tuyaux de conduite du faisceau, les alimentations de hautes tensions, les groupes rotatifs générant les courants continus de grande intensité circulant dans les aimants, le réseau de câbles reliant ces générateurs aux éléments de la machine, et, bien entendu, le pupitre de commande de l'accélérateur. 
Enfin, en avril 1965, le cyclotron à énergie variable accélère pour la première fois un faisceau interne de carbone quatre fois chargé $\left(\mathrm{C}^{4+}\right)$ jusqu'au rayon maximum de la machine, ce qui correspond à une énergie d'environ $90 \mathrm{MeV}^{8}$. Sans perdre de temps, Marc Lefort place à ce rayon une cible de platine et peut constater, après séparation chimique et mesure de radioactivité alpha, que des isotopes de polonium (Po) ont bien été produits par des réactions de fusion telles que : ${ }^{12} \mathrm{C}+{ }^{194} \mathrm{Pt}$ 䦪 ${ }^{206-\mathrm{x}} \mathrm{Po}+\mathrm{x} \mathrm{n}$, où le symbole $\mathrm{n}$ représente le neutron, et $\mathrm{x}$ le nombre de neutrons émis. Cette expérience, facile à mettre en œuvre, permet de concrétiser par une publication de physique ${ }^{9} \mathrm{le}$ démarrage, tant attendu, de l'accélérateur, et de démontrer son bon fonctionnement.

Il faudra attendre encore une année pour que soient résolus tous les problèmes liés à l'extraction du faisceau et que celui-ci puisse être envoyé dans les aires expérimentales. Trois postes sont alors à la disposition des physiciens. La sortie du canal B, sans équipement spécial, est réservée aux expériences d'irradiations de cibles ${ }^{10}$. Le canal $\mathrm{C}$ débouche sur une chambre à réaction de 1 mètre de diamètre, fonctionnant sous vide, et destinée à accueillir des détecteurs placés à différents angles ${ }^{11}$. Un effort d'équipement supérieur a été réalisé pour le canal $\mathrm{D}$ où un gros analyseur magnétique, identique, dans son principe, à celui qui équipe le synchrocyclotron [3], et placé en regard d'une grande chambre à réaction, permettra de réaliser des expériences avec une haute résolution en énergie.

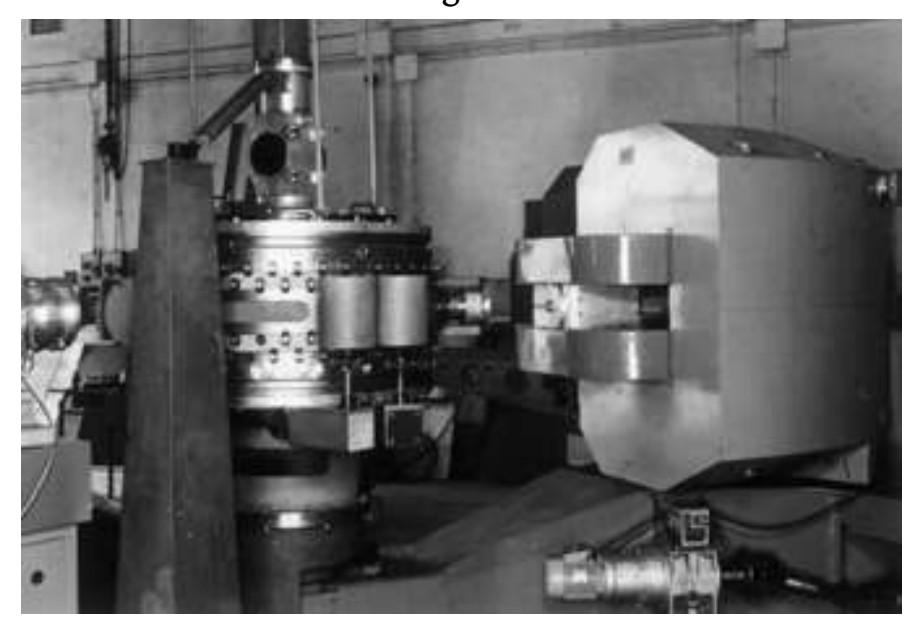

Le cyclotron à énergie variable. On remarque l'imposante masse rectangulaire de la culasse de l'électroaimant, qui englobe deux bobines cylindriques entre lesquelles est placée la chambre d'accélération. À gauche, la ligne résonante du générateur haute fréquence. () IPN 


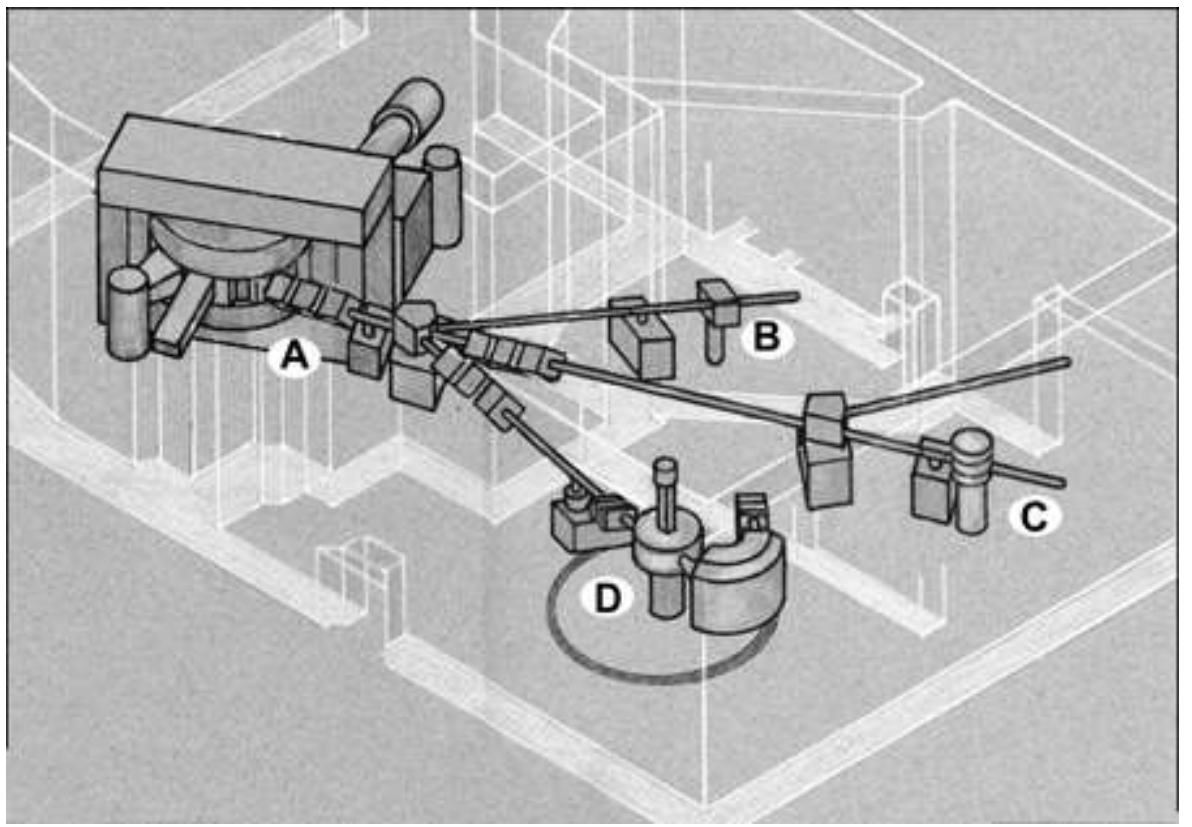

Implantation du CEV. Les ions extraits de la machine sont d'abord La chambre à réaction du canal C. envoyés dans une courte ligne de faisceau, le canal A, à la sortie duquel un aiguilleur magnétique permet de les distribuer alternativement dans trois autres canaux menant à trois postes expérimentaux, repérés par les lettres B, C et D. Schéma : A. Steinegger, @ IPN

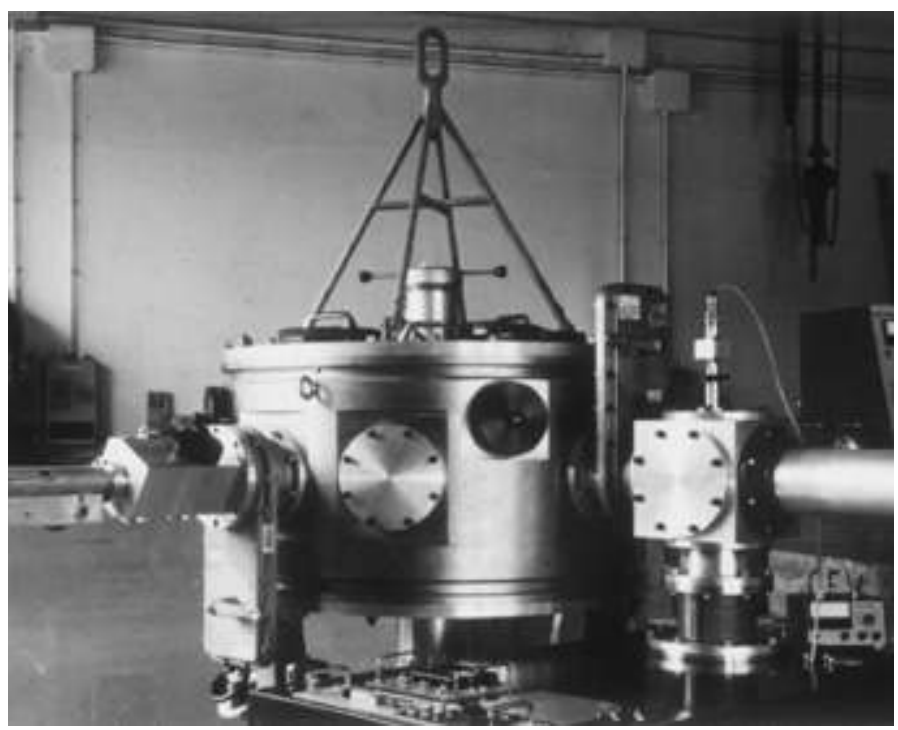

La chambre à réaction du canal C. (C) IPN 


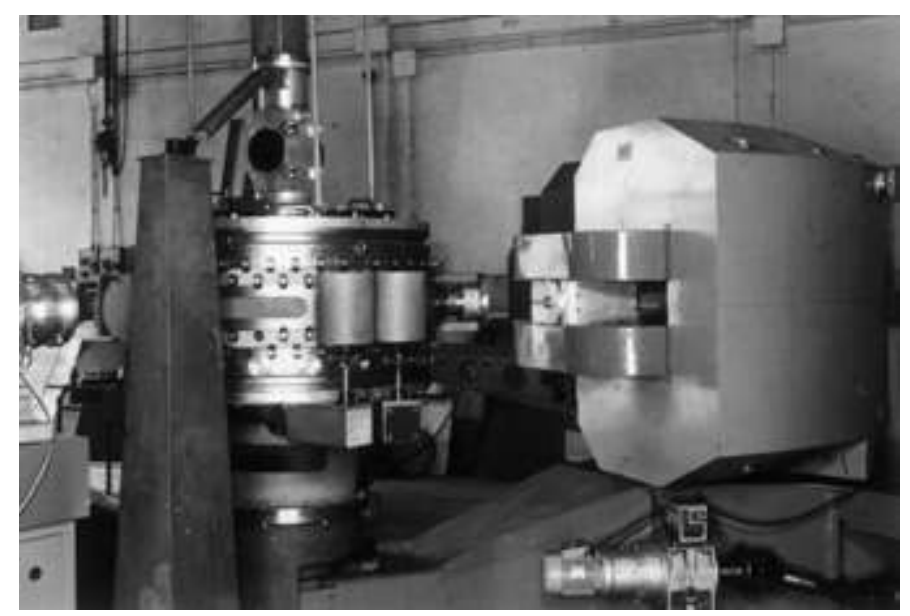

La chambre à réaction du canal D. (C) IPN

Les premières expériences

11 Le premier cyclotron français conçu pour les ions lourds [4, 5], est mis à la disposition des expérimentateurs à l'automne 1966. Malgré l'enthousiasme et l'imagination qui ont présidé à la réalisation de cette machine, qui se caractérise par de nombreuses innovations technologiques, les faisceaux de carbone et d'azote qu'elle délivre n'ont aucun caractère original. Ils sont comparables à ceux dont disposaient Américains et Soviétiques dix ans plus tôt, et surclassés par les performances de plusieurs accélérateurs, notamment ceux de Berkeley, Yale et Dubna (URSS). De plus, le fonctionnement du CEV est encore très incertain. Les intensités sont faibles et de nombreuses pannes viennent interrompre fréquemment les prises de données. Il en résulte qu'un temps non négligeable doit être consacré à améliorer le fonctionnement de la machine, et notamment celui des sources d'ions. En dix années, les principales caractéristiques des réactions induites par ions lourds ont été explorées et publiées. La situation des premiers utilisateurs du CEV n'est donc pas favorable. Ils tentent de surmonter ce handicap en réalisant, dès 1966, des expériences mettant en jeu des détecteurs performants et une électronique de pointe, de façon à affiner les résultats obtenus par leurs prédécesseurs, et à produire des bases de données plus précises, plus quantitatives. Les recherches sont ainsi lancées dans plusieurs directions.

L'étude des transferts d'un ou de plusieurs nucléons, du projectile vers la cible ou vice versa, est menée en parallèle par deux techniques. La première (équipe Riou-Jacmart) utilise l'aimant d'analyse du canal D ${ }^{12}$, la seconde (équipe Lefort-Tarrago), des télescopes de détecteurs solides ${ }^{13}$ montés dans la chambre à réaction du canal $\mathrm{C}{ }^{14}$. Toutes deux offrent des mesures d'énergie de bonne résolution, adaptées aux réactions étudiées, qui conduisent souvent à des niveaux d'énergie bien définis des noyaux produits, donc à des pics étroits dans les distributions en énergie des ions observés.

Des détecteurs solides placés dans la chambre à réaction du canal C sont également utilisés pour observer les particules légères (protons, deutons, particules alpha...) issues de la désexcitation de noyaux de fusion disposant de grandes énergies de rotation ${ }^{15}$. Des expériences d'irradiations de cibles avec mesures de la radioactivité des noyaux résiduels issus de l'évaporation de ces particules étudient le même phénomène dans une approche complémentaire ${ }^{16}$.

14 Enfin, des études de spectroscopie nucléaire sont entreprises en analysant les rayonnements gamma issus de l'excitation coulombienne de noyaux par des faisceaux 
d'azote ${ }^{17}$, ou en mesurant ceux qui sont émis en fin de chaîne lors de la désexcitation de noyaux composés. Dans les deux cas, l'énergie de ces rayonnements gamma est mesurée par des détecteurs à semi-conducteurs (Germanium-Lithium), dont l'utilisation commence tout juste à se répandre dans les laboratoires [6], et dont l'excellente résolution garantit la précision des mesures effectuées.

La Plagne : une réunion tournée vers l'avenir

À la première « Conférence sur les réactions entre noyaux complexes » avait succédé la seconde, également tenue à Gatlinburg, en 1960, puis la troisième, à Asilomar (Californie) en 1963. À Dubna, en URSS, une Conférence internationale sur les réactions par ions lourds avait été organisée en 1966. En France, le démarrage du cyclotron à énergie variable avait été préparé par trois journées d'études à Bordeaux, en 1964.

Mais la réunion qui marque vraiment l'entrée de notre pays dans la physique des ions lourds est celle qui se tient en février 1969 dans la toute nouvelle station alpine de La Plagne. Cette rencontre [7] a lieu un peu plus de deux années après la mise en service $\mathrm{du} \mathrm{CEV}$, et seuls quelques résultats parmi les premiers obtenus auprès de cette machine y sont présentés. En revanche, une large place y est donnée à la théorie, et à la technique des accélérateurs. Manifestement, cette session d'études est tournée vers l'avenir. En effet, l'Institut de physique nucléaire prépare un joker susceptible de modifier la donne internationale en ce qui concerne la physique des ions lourds. Il s'agit d'un petit accélérateur destiné à jouer le rôle d'injecteur pour le cyclotron à énergie variable. Ce couplage de deux machines différentes, qui n'a jamais été réalisé auparavant, doit permettre d'utiliser toute la versatilité du cyclotron en accélérant une large gamme d'ions lourds, et de surclasser les performances des accélérateurs américains et soviétiques. L'ensemble "accélérateur linéaire + cyclotron à énergie variable » portera le nom d'ALICE, et c'est grâce à lui que devrait s'opérer, en France, la véritable percée de la physique des ions lourds. Les chercheurs français n'auront pas trop à attendre pour savourer ce brillant retournement de situation [8].

\section{BIBLIOGRAPHIE}

- [1] Proceedings of the "Conference on reactions between complex nuclei", Gatlinburg, Tennessee, May 5-7, 1958, Rapport ORNL - 2606, Oak-Ridge National Laboratory (1958).

•[2] « Le cyclotron à énergie variable pour l'accélération d'ions lourds. Projet et études ». Publication interne du Laboratoire de physique nucléaire d'Orsay (1960), Archives de l'Institut de physique nucléaire d'Orsay.

-[3] Bimbot R. « Les années 'Synchro' (1958-1966) » in La revue pour l'histoire du CNRS, nº17, été 2007, pp. 40-45.

-[4] Cabrespine A. Le cyclotron à énergie variable d'Orsay. Industries atomiques, 11 (1966) 51.

•[5] Lefort M. Le programme scientifique du CEVIL d'Orsay. Industries atomiques, 11 (1966) 41. 
- [6] Bimbot R., Lavergne L. « IPN : l'émergence des détecteurs à semi-conducteurs » in La revue pour l'histoire du CNRS, n¹9, hiver 2007, pp. 44-48.

- [7] Compte-rendu de la session d'études « Sur la physique et la production des ions lourds », La Plagne 17-21 mars 1969, Rapport lycen 6913, Institut de physique nucléaire de Lyon, Villeurbanne (1969).

- [8] Bimbot R. « ALICE ou l'essor des ions lourds ». Vidéo de 28' réalisée par D. Garabédian (ENS Production) et S. Guyon (SAV, Orsay), distribuée par le SFRS, Paris (1989).

\section{NOTES}

1.Curieusement, c'est Luis Alvarez, dont le nom est resté attaché aux accélérateurs linéaires qui réalisa cette première sur le cyclotron de 88 pouces de Berkeley. Alvarez L. W. Phys. Rev. 58 (1940) 192.

2.Les dimensions, données en pouces ou en centimètres pour les cyclotrons, se rapportent au diamètre des pôles de leur aimant.

3.Son « jumeau » de Yale est alors en fin de construction.

4.Des ions lourds sont également accélérés dans les cyclotrons de Berkeley (États-Unis), Birmingham (Grande-Bretagne), et Stockholm (Suède).

5.De fait, de telles réactions viennent d'être utilisées (en 1957-58) par l'équipe de G. Seaborg et A. Ghiorso, à Berkeley, pour découvrir les éléments 102 et 103, qui seront baptisés ultérieurement nobélium et lawrencium, en bombardant des cibles (radioactives) de curium 246 (de numéro atomique $\mathrm{Z}=96$ ) avec des faisceaux de carbone $12(\mathrm{Z}=6)$ et d'azote $14(\mathrm{Z}=7)$.

6.L'intérêt théorique de ces réactions par ions lourds provient notamment du fait qu'elles peuvent s'interpréter dans une approche, dite " semi classique », qui combine la mécanique classique (existence de trajectoires bien définies) et la mécanique quantique, qui régit notamment le transfert de nucléons d'un partenaire à l'autre lors des collisions tangentielles. Voir M. Demeur « Discours de clôture », C. R. Journées d'études sur les réactions nucléaires par ions lourds, Bordeaux 16-18 janvier 1964, édité par le Laboratoire de physique nucléaire de la faculté des sciences de Bordeaux (1964). 7.Il s'agissait de créer des secteurs de champ fort et de champ faible, nécessaires pour maintenir une focalisation du faisceau en cours d'accélération.

8. $\mathrm{MeV}$ = million d'électron-Volts.

9.Brun C., Le Beyec Y., Lefort M. Isotopes légers du polonium produits par réactions nucléaires par ions de carbone 12 sur le platine. C. R. Acad. Sci. 261 (1965) 1667.

10.Afin de simplifier la mesure du nombre d'ions atteignant ces cibles, celles-ci seront le plus souvent placées au fond d'un cylindre de Faraday permettant de déterminer directement la charge électrique collectée pendant une irradiation donnée, d'où le nombre d'ions, connaissant la charge de chacun d'entre eux.

11.Un embranchement avait été prévu sur le canal $C$ pour équiper une ligne supplémentaire. De fait, il ne fut pas utilisé, une autre solution ayant été adoptée ultérieurement pour accroître le nombre de postes expérimentaux.

12.Mazloum F., Thèse de $3^{\mathrm{e}}$ cycle, Orsay (1968).

13. Cette technique est explicitée dans la référence [5].

14.Galin J. et al. Phys. Rev. 182 (1969) 1267.

15.Brun C. et al. Nucl. Phys. A116 (1968) 177. 
16.Bimbot R. et al. J. Phys. 29 (1968) 563.

17.Sergolle H. et al. Nucl. Phys. A145 (1970) 351.

\section{RÉSUMÉS}

En 1958, se tient à Gatlinburg, Tennessee, la première conférence internationale sur les réactions nucléaires induites par ions lourds, mais ce n'est qu'en 1969, à La Plagne, qu'une grande réunion scientifique française a lieu sur ce thème. Les onze années qui séparent ces deux dates ont été mises à profit par le tout jeune Laboratoire de physique nucléaire d'Orsay pour construire, par ses propres moyens, un cyclotron à ions lourds, et réaliser ses premières expériences. C'est cette session d'études de La Plagne qui donnera le véritable coup d'envoi à la physique des ions lourds en France.

In 1958, the first international meeting on heavy ion reactions is organized at Gatlinburg, Tennessee, but it is only in 1969 that the first French important workshop dealing with this theme takes place at La Plagne. During the eleven years which separate these two dates, the Orsay 'Laboratoire de physique nucléaire' has built by itself a heavy ion cyclotron and performed its first experiments. The La Plagne meeting prefigures the future break through of French heavy ion physics.

\section{AUTEUR}

\section{RENÉ BIMBOT}

René Bimbot est agrégé de physique, docteur ès sciences, directeur de recherche émérite à l'IPN et au CNRS. 